\title{
Limits on the neutrino mass and mixing angle from pion and lepton decays
}

\author{
A. Bottino,,$^{1,2}{ }^{*}$ N. Fornengo, ${ }^{2,3, \dagger}$ C. W. Kim, ${ }^{3, \dagger}$ and G. Mignola ${ }^{1,2, \S}$ \\ ${ }^{1}$ Dipartimento di Fisica Teorica, Università di Torino, Via P. Giuria 1, 10125 Torino, Italy \\ ${ }^{2}$ INFN, Sezione di Torino, Via P. Giuria 1, 10125 Torino, Italy \\ ${ }^{3}$ Department of Physics and Astronomy, The Johns Hopkins University, Baltimore, Maryland 21218
}

(Received 26 May 1995; revised manuscript received 4 January 1996)

\begin{abstract}
Motivated by a recent rather surprising conclusion based on the 1992 PDG data on the pion, kaon, and lepton decays that if three generations of neutrinos are assumed to be massive and mixed, the heaviest neutrino $\nu_{3}$ could have a mass in the range $155 \mathrm{MeV} \lesssim m_{3} \lesssim 225 \mathrm{MeV}$, we have analyzed the latest 1995 data on the leptonic decays of the pion, $\mu$ and $\tau$ with the assumption that three generations of neutrinos are massive and mixed. It is shown that when the radiative corrections are included and the constraint from partial decay widths is imposed, the 1995 data are consistent with three massless neutrinos with no mixing. Various limits on the neutrino mass and mixing angle implied by the 1995 data are presented together with a critique of the previous analysis. [S0556-2821(96)06111-5]

PACS number(s): 14.60.Pq, 13.35.-r
\end{abstract}

\section{INTRODUCTION}

In a series of seminal papers [1] in the early 1980s, Shrock proposed a wide range of experimental methods to obtain possible limits on the neutrino mass and associated mixing, all based on a precision analysis of weak interaction data on the decays of the pion, kaon, and charged leptons $\mu$ and $\tau$. In these papers, a comprehensive analysis of decay rates and branching ratios of the lepton and meson decays was carried out using a theoretical framework with three massive neutrinos and associated mixing. (It is interesting to note that as early as in 1961 Bahcall and Curtis [2] proposed a similar method based on pion and muon decays, even before the discovery of $\nu_{\mu}$.) At that time, however, available data were not accurate enough to provide any significant results on the limits on the neutrino mass and mixing angle, in the sense that the limits on the mixing angles were restricted mostly for large values of the neutrino masses. In the later works $[3,4]$ the limits were further improved.

The best known and often quoted limits on the neutrino mass still come from the analysis of spectral shapes in the Kurie plots or other decay kinematics [5]:

$$
\begin{gathered}
m_{1} \equiv m\left(\nu_{1}\right) \lesssim 5 \mathrm{eV} \quad[4], \\
m_{2} \equiv m\left(\nu_{2}\right) \lesssim 270 \mathrm{keV} \quad[4], \\
m_{3} \equiv m\left(\nu_{3}\right) \lesssim 24 \mathrm{MeV} \quad[6] .
\end{gathered}
$$

The results from these analyses are always presented with the assumption of neutrinos with no mixing. A full analysis of the spectral shapes with three massive neutrinos with mixing is very much involved and so far no such analysis with satisfactory accuracy has been carried out.

\footnotetext{
*Electronic address: bottino@to.infn.it

†Electronic address: fornengo@jhup.pha.jhu.edu

¥Electronic address: kim@ rowland.pha.jhu.edu

${ }^{\S}$ Electronic address: mignola@to.infn.it
}

In the meantime, much attention has been focused on entirely different approaches in which neutrino mass and associated mixing can be probed indirectly by searching for neutrino oscillation phenomena. Recent activities in this approach include the experimental search for reactor and accelerator neutrino oscillations and the study of solar and atmospheric neutrinos. Although very intriguing indications of massive neutrinos with mixing have recently been hinted at in solar and atmospheric neutrino experiments and in the LSND experiment, a definitive answer from these experiments is yet to come.

Recently, Peres, Pleitez, and Zukanovich Funchal (PPZ) [7] carried out a comprehensive analysis of the existing data on meson and lepton weak decays assuming that three generations of neutrinos are massive and mixed. Their analysis was based on the 1992 Particle Data Group (PDG) data [8] combined with the latest (in 1993) data on $\tau$ decays [9]. The results are quite surprising in that the 1992 data on the decay branching ratios were consistent with a finite mass for $\nu_{3}$, i.e., $155 \mathrm{MeV} \lesssim m_{3} \lesssim 225 \mathrm{MeV}$. This mass range is significantly larger than the most recent upper limit $m_{3} \leqslant 24 \mathrm{MeV}$ that was obtained from a kinematical analysis of the $\tau$ decay into five or six pions and $\nu_{\tau}$ with no mixing. Moreover, PPZ found that the mixing angle $\beta$ which represents mixing between $\nu_{1}$ and $\nu_{3}$ is also finite $\left(11^{\circ}-12^{\circ}\right)$ whereas the mixing angle $\gamma$ between $\nu_{2}$ and $\nu_{3}$ was bounded from above, thus allowing a zero mixing angle.

Motivated by these rather surprising results, we have carried out a similar analysis of the decay rates and branching ratios of the leptonic decays of the pion, $\mu$, and $\tau$ with the assumption of massive neutrinos with mixing. First we have repeated the PPZ analysis with the same set of data (1992 PDG data) and with the assumptions used by PPZ, confirming their results. However, we have also found that their results are significantly modified when the constraint coming from the partial decay rates, which PPZ did not use, is imposed. The constraint imposed by the decay rate is not an independent one. Instead, it ensures that possible fortuitous cancellations in the ratios will not lead to erroneous conclusions. Furthermore, we have found that the radiative correc- 
tions, which PPZ also ignored, are quite important because the accuracy of the data more than warrants the inclusion of the radiative corrections in a precision analysis such as this. A similar analysis using the latest 1995 data shows that the 1995 data are consistent with the picture of three massless neutrinos with no mixing. We have been able to set various limits on the neutrino masses and mixing angles.

The plan of our paper is as follows. In Sec. II, we list the formulas relevant to our analysis. (Some details are put in the Appendix.) All the data used in our analysis are collected in Sec. III, including the data of 1992 for comparison. In Sec. IV, we present details on our reexamination and critique of the PPZ analysis. In particular, we discuss here what would happen to the PPZ conclusions based on the 1992 PDG data, when the radiative corrections are included and the con-

$$
V=\left(\begin{array}{c}
c_{\theta} c_{\beta} \\
-s_{\theta} c_{\gamma}-c_{\theta} s_{\gamma} s_{\beta} \\
s_{\theta} s_{\gamma}-c_{\theta} c_{\gamma} s_{\beta}
\end{array}\right.
$$

where $s_{\theta} \equiv \sin \theta, c_{\theta} \equiv \cos \theta, s_{\beta} \equiv \sin \beta, \ldots$. In Eq. (3), the angle $\theta$ refers to mixing between $\nu_{1}$ and $\nu_{2}, \beta$ to $\nu_{1}$ and $\nu_{3}$, and $\gamma$ to $\nu_{2}$ and $\nu_{3}$, respectively.

In our analysis, we will discuss the limits on neutrino masses and mixing angles which can be inferred from purely leptonic decays of pion and leptons ( $\mu$ and $\tau$ ). That is, the decay rates to be used are

$$
\begin{gathered}
\Gamma\left(\pi \rightarrow e \bar{\nu}_{e}\right), \quad \Gamma\left(\pi \rightarrow \mu \bar{\nu}_{\mu}\right), \\
\Gamma\left(\mu \rightarrow e \bar{\nu}_{e} \nu_{\mu}\right), \\
\Gamma\left(\tau \rightarrow e \bar{\nu}_{e} \nu_{\tau}\right), \quad \Gamma\left(\tau \rightarrow \mu \bar{\nu}_{\mu} \nu_{\tau}\right) .
\end{gathered}
$$

These are the best known experimental quantities which do not involve hadrons in the final states, hence introducing no further unnecessary complications in the calculation of decay widths. We will not consider the $K$ decays, even though some experimental determinations of its decay widths into leptons are almost as good as those of the pion. Its properties are quite similar to those of the pion and its data do not provide any additional (or critical) information.

Here, we briefly summarize the formulas to be used. (Details are given in the Appendix.) For the pion, the decay rate into two leptons in a general case of three massive neutrinos with mixing is given by

$$
\Gamma\left(\pi \rightarrow l \bar{\nu}_{l}\right)=\frac{G^{2} f_{\pi}^{2} U_{u d}^{2} m_{\pi}^{3}}{8 \pi} \mathcal{R}_{\pi l} \sum_{i=1}^{3}\left|V_{l i}\right|^{2} P_{i}^{\pi l}
$$

where $G$ is the Fermi constant (see comment below), $m_{\pi}$ is the pion mass, $f_{\pi}$ is the pion decay constant, and $U_{u d}$ is the $u d$ component of the mixing matrix in the quark sector. The matrix-element-phase-space function $P_{i}^{\pi l}$ denotes the quantity of our interest which contains part of the matrix element straint from the partial decay rates is imposed. New results based on the 1995 data are presented in Sec. V, and a summary and conclusions are given in Sec. VI.

\section{FORMULAS WITH THREE GENERATION MIXING}

The mixing matrix $V$ in the lepton sector which relates the (weak) interaction eigenfields $\nu_{\alpha}(\alpha=e, \mu, \tau)$ to the mass eigenfields $\nu_{i}(i=1,2,3)$ is given by

$$
\nu_{\alpha}=\sum_{i=1}^{3} V_{\alpha i} \nu_{i}
$$

We parametrize the mixing matrix $V$ using the Maiani representation [10] of the mixing matrix $U$ in the quark sector, with the $C P$-violating phase set to zero: i.e.,

$$
\left.\begin{array}{cc}
s_{\theta} c_{\beta} & s_{\beta} \\
c_{\theta} c_{\gamma}-s_{\theta} s_{\gamma} s_{\beta} & s_{\gamma} c_{\beta} \\
c_{\theta} s_{\gamma}-s_{\theta} c_{\gamma} s_{\beta} & c_{\gamma} c_{\beta}
\end{array}\right) .
$$

and the entire phase space, and it depends on the neutrino masses $m_{i}(i=1,2,3)$, as well as on the pion and lepton masses. $\mathcal{R}_{\pi l}$ is a factor that represents the radiative corrections to the process. We stress here that $\mathcal{R}_{\pi l}$ depends on the pion mass as well as on the lepton mass. The complete expressions for $P_{i}^{\pi l}$ and $\mathcal{R}_{\pi l}$ are given in the Appendix. We wish to emphasize here that the value of $f_{\pi}=(130.7 \pm 0.1 \pm 0.36) \mathrm{MeV}$ quoted in the PDG data set is obtained from the decay $\pi \rightarrow \mu \bar{\nu}_{\mu}+\mu \bar{\nu}_{\mu} \gamma$ under the hypothesis that the neutrinos are massless with no mixing. For massive and mixed neutrinos, the above value represents the quantity $\left[f_{\pi}^{2} \sum_{i=1}^{3}\left|V_{\mu i}\right|^{2} P_{i}^{\pi \mu} / P_{0}^{\pi \mu}\right]^{1 / 2}$ rather than $f_{\pi}$, where $P_{0}^{\pi \mu}$ is the matrix-element-phase-space function for massless neutrinos. For this reason, in the following, we will consider, as was done by PPZ, only the ratio of the two leptonic decay widths of the pion, in order to cancel out the dependence on the unknown quantity $f_{\pi}$.

The decay width for a lepton decaying into three leptons is given by

$$
\Gamma\left(l^{\prime} \rightarrow l \bar{\nu}_{l} \nu_{l^{\prime}}\right)=\frac{G^{2} m_{l^{\prime}}^{5}}{192 \pi^{3}} \mathcal{R}_{l^{\prime}} \sum_{i, j=1}^{3}\left|V_{l^{\prime} i}\right|^{2}\left|V_{l j}\right|^{2} P_{i j}^{l^{\prime} l},
$$

where $m_{l^{\prime}}$ is the mass of the decaying particle. Again, $P_{i j}^{l^{\prime} l}$ is the matrix-element-phase-space function which depends on the masses of all the particles involved in the decay process. The leading radiative corrections are denoted by $\mathcal{R}_{l^{\prime}}$; they depend only on the mass of the decaying lepton. Also, the expressions for $P_{i j}^{l^{\prime} l}$ and $\mathcal{R}_{l^{\prime}}$ are collected in the Appendix.

Again, it is crucial to emphasize, as noted by PPZ, that the experimental value of $G_{\mu}$ quoted in the PDG data set becomes the Fermi constant $G$ only for the massless neutrinos, 
as implied by the standard model. Since new physics beyond the standard model is what we wish to investigate, the coupling constant $G_{\mu}$ that one measures in the muon decay should be interpreted as

$$
\begin{aligned}
G_{\mu}^{2}= & {\left[\frac{G^{2}}{P_{00}^{\mu e}}\right] \sum_{i, j=1}^{3}\left|V_{\mu i}\right|^{2}\left|V_{e j}\right|^{2} P_{i j}^{\mu e} } \\
= & {\left[\frac{G^{2}}{P_{00}^{\mu e}}\right]\left\{c_{\theta}^{2} c_{\beta}^{2}\left(s_{\theta} c_{\gamma}+c_{\theta} s_{\gamma} s_{\beta}\right)^{2} P_{11}^{\mu e}+c_{\beta}^{2}\left[c_{\theta}^{2}\left(c_{\theta} c_{\gamma}+s_{\theta} s_{\gamma} s_{\beta}\right)^{2}+s_{\theta}^{2}\left(s_{\theta} c_{\gamma}+c_{\theta} s_{\gamma} s_{\beta}\right)^{2}\right] P_{12}^{\mu e}\right.} \\
& +\left[c_{\beta}^{2}\left(c_{\theta}^{2} c_{\beta}^{2} s_{\gamma}^{2}\right)+s_{\beta}^{2}\left(s_{\theta} c_{\gamma}+c_{\theta} s_{\gamma} s_{\beta}\right)^{2}\right] P_{13}^{\mu e}+s_{\theta}^{2} c_{\beta}^{2}\left(c_{\theta} c_{\gamma}+s_{\theta} s_{\gamma} s_{\beta}\right)^{2} P_{22}^{\mu e} \\
& \left.+\left[c_{\beta}^{2}\left(s_{\theta}^{2} c_{\beta}^{2} s_{\gamma}^{2}\right)+s_{\beta}^{2}\left(c_{\theta} c_{\gamma}+s_{\theta} s_{\gamma} s_{\beta}\right)^{2}\right] P_{23}^{\mu e}+s_{\beta}^{2} c_{\beta}^{2} s_{\gamma}^{2} P_{33}^{\mu e}\right\} \rightarrow G^{2} \text { as } m_{1}, m_{2}, m_{3} \rightarrow 0,
\end{aligned}
$$

where $P_{00}^{\mu e}$ is the matrix-element-phase-space function for massless neutrinos. Therefore, in the general case of massive neutrinos with mixing, the weak coupling constant $G$, which enters in the calculation of all the weak processes such as, e.g., Eqs. (4) and (5), is not directly measured from the muon decay. The quoted number $G_{\mu}=(1.16639 \pm 0.00002)$ $\times 10^{-5} \mathrm{GeV}^{-2}$ is valid only for massless neutrinos.

Obviously, a way to obtain the correct value of $G$ for massive and mixed neutrinos is to use Eq. (6). That is, from the known value of $G_{\mu}$ and the calculated phase-space factors in the above equation, $G$ can be derived for each value of the neutrino mass and mixing angle. The accuracy of the calculated value of $G$ is the same as that of $G_{\mu}$. This is the right procedure to be adopted in the calculation of weak processes if the hypothesis of massive neutrinos is assumed. However, as far as the present analysis is concerned, this procedure has the disadvantage of introducing the phasespace factor of the muon decay in the calculation of all the other decay widths, making them equivalent to the ratios $\Gamma\left(\tau \rightarrow e \nu_{\tau} \bar{\nu}_{e}\right) / \Gamma\left(\mu \rightarrow e \nu_{\mu} \bar{\nu}_{e}\right)$ and $\Gamma\left(\tau \rightarrow \mu \nu_{\tau} \bar{\nu}_{\mu}\right) /$ $\Gamma\left(\mu \rightarrow e \nu_{\mu} \bar{\nu}_{e}\right)$. Since, as we will show in the following, the use of the ratios alone can give overestimated allowed regions for the neutrino parameters due to fortuitous cancellations in the numerator and the denominator, we choose not to adopt this procedure.

An alternative way to obtain $G$ comes from the standard model of electroweak interactions, where $G$, the finestructure constant $\alpha$, the $W$ boson mass $m_{W}$, and the $Z$ boson mass $m_{Z}$ in the on-shell scheme of renormalization are related as [11]

$$
G=\frac{\pi \alpha}{\sqrt{2} m_{W}^{2}\left(1-m_{W}^{2} / m_{Z}^{2}\right)(1-\Delta r)} .
$$

The above equation relates the low-energy effective coupling constant $G$ of the weak interactions to the fine-structure constant and the weak bosons masses. Radiative effects, due to loop contributions of the fermions and the Higgs boson, are taken into account in $\Delta r$. The actual form of the radiative correction $\Delta r$ is given in Ref. [11]. Hence, our approach is to use Eq. (7) in order to calculate the value and the allowed $1 \sigma$ range for $G$, using $\alpha, m_{W}$, and $m_{Z}$ as input parameters. Obviously we expect the accuracy of the value of $G$ to be rather poor, i.e., of the same order of the experimental error on $m_{W}$. It is encouraging, however, that the latest measurements on the $W$ mass are at the level of 2\% [12]. Some additional input parameters, which enter in the radiative corrections $\Delta r$, are the mass of the top quark $m_{t}$ and the Higgs boson mass. For the top quark mass, we use the recent Collider Detector at Fermilab (CDF) Collaboration measurement $m_{t}=(176 \pm 18) \mathrm{GeV},[13]$ both for the 1992 and 1995 data sets. The Higgs boson mass is varied in the interval $(60 \mathrm{GeV}$, $1 \mathrm{TeV})$.

In the case of the 1992 data set, we use the 1992 PDG values for $m_{Z}$ and $m_{W}$. The result is (here and hereafter, the errors are propagated quadratically)

$$
G=(1.162 \pm 0.029) \times 10^{-5} \mathrm{GeV}^{-2}
$$

For the 1995 data, in order to reduce as much as possible the uncertainties in the determination of $G$, we use the latest data available: $m_{Z}=(91.1884 \pm 0.0022) \mathrm{GeV} \quad[14]$ and $m_{W}=(80.410 \pm 0.180) \mathrm{GeV}$ [12]. This gives

$$
G=(1.174 \pm 0.022) \times 10^{-5} \mathrm{GeV}^{-2}
$$

The values of $G$ which we will use in the evaluation of the leptonic decay widths are those given in the two previous equations. The errors on $G$ (of the order of percent) are much worse than the errors on $G_{\mu}$ which are of the order of $10^{-5}$. Nevertheless, as will be seen in the following, it is still possible to use the calculation of the decay widths as an important constraint on the neutrino parameters (mainly on the neutrino masses).

Measured experimentally are the branching ratios of the above decay processes. The branching ratios are simply related to the previously defined quantities as

$$
B\left(\pi \rightarrow l \bar{\nu}_{l}\right)=\frac{\Gamma\left(\pi \rightarrow l \bar{\nu}_{l}\right)}{\Gamma_{\pi}}=\tau_{\pi} \Gamma\left(\pi \rightarrow l \bar{\nu}_{l}\right)
$$




$$
B\left(l^{\prime} \rightarrow l \bar{\nu}_{l} \nu_{l^{\prime}}\right)=\frac{\Gamma\left(l^{\prime} \rightarrow l \bar{\nu}_{l} \nu_{l^{\prime}}\right)}{\Gamma_{l^{\prime}}}=\tau_{l^{\prime}} \Gamma\left(l^{\prime} \rightarrow l \bar{\nu}_{l} \nu_{l^{\prime}}\right),
$$

where $\Gamma_{\pi}$ and $\Gamma_{l^{\prime}}$ are the total widths of the pion and the decaying lepton, respectively, and $\tau_{\pi}$ and $\tau_{l^{\prime}}$ are the corresponding lifetimes. In order to directly extract information about the neutrino mass and mixing, instead of the lepton decay widths themselves, we use the following quantities which are simply proportional to the decay widths, with common constants such as $G$ and $m_{l}$, removed:

$$
\bar{\Gamma}^{l^{\prime} l}=\alpha_{l^{\prime}} B\left(l^{\prime} \rightarrow l \overline{\nu_{l}} \nu_{l^{\prime}}\right)=\mathcal{R}_{l^{\prime}} \sum_{i, j=1}^{3}\left|V_{l^{\prime}}\right|^{2}\left|V_{l j}\right|^{2} P_{i j}^{l^{\prime} l}
$$

where

$$
\alpha_{l^{\prime}}=\frac{192 \pi^{3}}{G^{2} m_{l^{\prime}}^{5} \tau_{l^{\prime}}}
$$

From the experimental values of the branching ratios (BR's) and the physical quantities defined in Eq. (13), we will first calculate the $1 \sigma$ allowed experimental ranges for the $\bar{\Gamma}$ 's. These ranges will then be compared with the calculated values of the $\bar{\Gamma}$ 's by varying the neutrino masses and mixing angles. This would limit the neutrino masses and mixing angles.

We will follow the same procedure for some ratios of the $\bar{\Gamma}$ 's and the BR's. Our choice of the ratios is

$$
\begin{aligned}
R_{\pi \mu}^{\pi e} & \equiv \frac{B\left(\pi \rightarrow e \overline{\nu_{e}}\right)}{B\left(\pi \rightarrow \mu \bar{\nu}_{\mu}\right)}=\frac{\mathcal{R}_{\pi e} \Sigma_{i}\left|V_{e i}\right|^{2} P_{i}^{\pi e}}{\mathcal{R}_{\pi \mu} \Sigma_{i}\left|V_{\mu i}\right|^{2} P_{i}^{\pi \mu}}, \\
R_{l_{2}^{\prime} l_{2}}^{l_{1}^{\prime} l_{1}} & \equiv \frac{\bar{\Gamma}^{l_{1}^{\prime} l_{1}}}{\overline{\Gamma_{2}^{\prime}}}=\left(\frac{m_{l_{2}^{\prime} l_{2}}^{5} \tau_{l_{2}^{\prime}}}{m_{l_{1}^{\prime}}^{5} \tau_{l_{1}^{\prime}}^{\prime}}\right) \frac{B\left(l_{1}^{\prime} \rightarrow l_{1} \bar{\nu}_{l_{1}} \nu_{l_{1}^{\prime}}\right)}{B\left(l_{2}^{\prime} \rightarrow l_{2} \bar{\nu}_{l_{2}} \nu_{l_{2}^{\prime}}\right)} \\
& =\frac{\mathcal{R}_{l_{1}^{\prime}} \Sigma_{i, j}\left|V_{l_{1}^{\prime} i}\right|^{2}\left|V_{l_{1} j}\right|^{2} P_{i j}^{l_{1}^{\prime} l_{1}}}{\mathcal{R}_{l_{2}^{\prime}} \Sigma_{i, j}\left|V_{l_{2}^{\prime} i}\right|^{2}\left|V_{l_{2} j}\right|^{2} P_{i j}^{l_{2}^{\prime} l_{2}}} .
\end{aligned}
$$

In the case of lepton decays, we will perform our analysis using the two ratios $R_{\tau e}^{\mu e}$ and $R_{\tau \mu}^{\tau e}$. The use of ratios alone is indeed simpler because uncertainties in some constant quantities are canceled out, but some changes in the numerator and the denominator coming from phase-space and mixing angles may partially be compensated. Therefore one must check that the calculated single partial decay widths do not lie outside the experimentally allowed ranges. This is why we will add as an additional constraint also the three leptonic decay widths. Summarizing, we will use the following quantities as constraint: $\bar{\Gamma}^{\mu e}, \bar{\Gamma}^{\tau e}, \bar{\Gamma}^{\tau \mu}, R_{\pi \mu}^{\pi e}, R_{\tau e}^{\mu e}$, and $R_{\tau \mu}^{\tau e}$.

When we evaluate the $\bar{\Gamma}$ 's and the $R$ 's from the experimental values, we propagate the errors quadratically. In the calculations, we use the central values of masses of the particles involved. All the calculations are carried out at the $1 \sigma$ level. Also, it is to be noted that the radiative correction factors $\mathcal{R}$ 's are included in the quantities $\bar{\Gamma}$ 's; they are canceled out in $R_{\tau \mu}^{\tau e}$ but not in $R_{\tau e}^{\mu e}$ and $R_{\pi \mu}^{\pi e}$.

\section{NUMERICAL INPUTS}

We have listed, in Table I, all the latest (1995 data) experimental inputs that will be used in our analysis $[4,15]$. In order to compare our new analysis with the previous one by PPZ based on the 1992 PDG data, we have also listed the 1992 PDG data [8] in Table I. As can be seen in Table I, the entries with asterisks signify those with noticeable changes from the 1992 data to the 1995 data. In particular, they include all the data on $\tau$ decays and $\pi \rightarrow e \bar{\nu}_{e}$. For the sake of comparison, in Table I the value of both $G_{\mu}$ and $G$ are included. (We note that, strictly speaking, $m_{\tau}$ quoted in Table I is for massless $\nu_{\tau}$. However, in this case, the use of $m_{\tau}$ in Table I does not introduce any significant modification.)

We should notice that the errors on the $\bar{\Gamma}$ 's are quite large (of the order of a few percent) due to the uncertainties in the Fermi constant $G$, as discussed in the previous section. Nevertheless, the inclusion of the $\bar{\Gamma}$ constraint turns out to be effective in limiting the allowed intervals for the neutrino parameters, in particular the masses, as will be shown in the following sections.

Listed below are the calculated values of $\bar{\Gamma}$ 's and $R$ 's using the latest mass values for the decaying particles and charged leptons under the assumption that neutrinos are massless with no mixing. In the case of $\bar{\Gamma}$ 's, the first numbers on the right-hand side are the values without the radiative corrections and the second numbers represent the radiative corrections. All the values are in agreement with the 1995 data in Table I within $1 \sigma$, implying that the 1995 data are consistent with the lepton sector with massless neutrinos with no mixing.

Calculated values for massless neutrinos with no mixing (1995 data set):

$$
\begin{gathered}
\bar{\Gamma}^{\mu e}=0.999813 \times 0.995797=0.995611, \\
R_{\pi \mu}^{\pi e}=1.233 \times 10^{-4}, \\
\bar{\Gamma}^{\tau e}=1.00 \times 0.996=0.996, \quad R_{\tau e}^{\mu e}=0.9995, \\
\bar{\Gamma}^{\tau \mu}=0.972 \times 0.996=0.968, \quad R_{\tau \mu}^{\tau e}=1.028 .
\end{gathered}
$$

(The uncertainties in the above numbers due to the experimental errors of the quantities which enter in their calculations are always less than $0.01 \%$.)

The conclusion based on the above numbers that the experimental data (1995) are consistent with the assumption of massless neutrinos with no mixing is in sharp contrast to the result coming from the 1992 PDG data, as obtained by PPZ. In order to further examine 1992 data set, we have repeated the calculations using the 1992 PDG data both with and without the inclusion of the radiative corrections (RC's). We list, in the following, the results of the calculated values based on the 1992 PDG data in Table I with the assumption that neutrinos are massless with no mixing.

Calculated values for massless neutrinos with no mixing (1992 PDG data set): 
TABLE I. List of 1992 and 1995 data sets. The entries with asterisks denote data which have been improved by a significant amount.

\begin{tabular}{|c|c|c|c|}
\hline & & 1992 data & 1995 data \\
\hline \multirow{5}{*}{$*$} & $m_{e}$ & $(0.51099906 \pm 0.00000015) \mathrm{MeV}$ & Same as 1992 \\
\hline & $m_{\mu}$ & $(105.658389 \pm 0.000034) \mathrm{MeV}$ & Same as 1992 \\
\hline & $m_{\tau}$ & $(1784.1 \pm 3.6) \mathrm{MeV}$ & $(1776.96 \pm 0.31) \mathrm{MeV}$ \\
\hline & $m_{\pi}$ & $(139.5679 \pm 0.0007) \mathrm{MeV}$ & $(139.56995 \pm 0.00035) \mathrm{MeV}$ \\
\hline & $\tau_{\mu}$ & $(2.19703 \pm 0.00004) \times 10^{-6} \mathrm{~s}$ & Same as 1992 \\
\hline$*$ & $\tau_{\tau}$ & $(305 \pm 6) \times 10^{-15} \mathrm{~s}$ & $(291.6 \pm 1.5) \times 10^{-15} \mathrm{~s}$ \\
\hline$*$ & $m_{Z}$ & $(91.173 \pm 0.020) \times 10^{3} \mathrm{MeV}$ & $(91.1884 \pm 0.0022) \times 10^{3} \mathrm{MeV}$ \\
\hline$*$ & $m_{W}$ & $(80.22 \pm 0.26) \times 10^{3} \mathrm{MeV}$ & $(80.410 \pm 0.180) \times 10^{3} \mathrm{MeV}$ \\
\hline & $G_{\mu}$ & $(1.16639 \pm 0.00002) \times 10^{-11} \mathrm{MeV}^{-2}$ & Same as 1992 \\
\hline * & $G$ & $(1.162 \pm 0.029) \times 10^{-11} \mathrm{MeV}^{-2}$ & $(1.174 \pm 0.022) \times 10^{-11} \mathrm{MeV}^{-2}$ \\
\hline$*$ & $B\left(\pi \rightarrow e \bar{\nu}_{e}\right)$ & $(1.218 \pm 0.014) \times 10^{-4}$ & $(1.230 \pm 0.004) \times 10^{-4}$ \\
\hline & $B\left(\pi \rightarrow \mu \bar{\nu}_{\mu}\right)$ & $0.9998782 \pm 0.0000014$ & $0.9998770 \pm 0.0000004$ \\
\hline & $B\left(\mu \rightarrow e \bar{\nu}_{e} \nu_{\mu}\right)$ & 1 & Same as 1992 \\
\hline$*$ & $B\left(\tau \rightarrow e \bar{\nu}_{e} \nu_{\tau}\right)$ & $0.1793 \pm 0.0026$ & $0.1779 \pm 0.0009$ \\
\hline$*$ & $B\left(\tau \rightarrow \mu \bar{\nu}_{\mu} \nu_{\tau}\right)$ & $0.1758 \pm 0.0027$ & $0.1733 \pm 0.0009$ \\
\hline$*$ & $\bar{\Gamma}^{\mu e}$ & $1.003 \pm 0.050$ & $0.983 \pm 0.037$ \\
\hline$*$ & $\bar{\Gamma}^{\tau e}$ & $0.944 \pm 0.053$ & $0.979 \pm 0.037$ \\
\hline$*$ & $\bar{\Gamma}^{\tau \mu}$ & $0.925 \pm 0.052$ & $0.954 \pm 0.036$ \\
\hline$*$ & $R_{\pi \mu}^{\pi e}$ & $(1.218 \pm 0.013) \times 10^{-4}$ & $(1.230 \pm 0.004) \times 10^{-4}$ \\
\hline * & $R_{\tau e}^{\mu e}$ & $1.063 \pm 0.028$ & $1.0038 \pm 0.0073$ \\
\hline$*$ & $R_{\tau \mu}^{\tau e}$ & $1.020 \pm 0.022$ & $1.0265 \pm 0.0074$ \\
\hline
\end{tabular}

$\bar{\Gamma}^{\mu e}=0.999813 \times 0.995797=0.995611$,

$$
\begin{gathered}
\bar{\Gamma}^{\tau e}=1.00 \times 0.996=0.996, \\
\bar{\Gamma}^{\tau \mu}=0.973 \times 0.996=0.969
\end{gathered}
$$

$$
\begin{array}{cl}
R_{\pi \mu}^{\pi e}=1.233 \times 10^{-4} & \left(R_{\pi \mu}^{\pi e}=1.283 \times 10^{-4} \text { without RC's }\right) \\
R_{\tau e}^{\mu e}=0.9995 & \left(R_{\tau e}^{\mu e}=0.9998 \text { without RC's }\right) \\
R_{\tau \mu}^{\tau e}=1.028 & \left(R_{\tau \mu}^{\tau e}=1.028 \text { without RC's }\right)
\end{array}
$$

(Again, the uncertainties in the above numbers are also less than $0.01 \%$.)

Note that the calculated values of the following quantities do not lie in their corresponding experimental $1 \sigma$ ranges: $R_{\pi \mu}^{\pi e}$ and $R_{\tau e}^{\mu e}$. This implies that the 1992 PDG data are indeed incompatible, at least within $1 \sigma$, with the assumption that neutrinos are massless with no mixing. If the radiative corrections are not included, $\bar{\Gamma}^{\tau e}$ is not compatible, also.

\section{REEXAMINATION OF PREVIOUS ANALYSIS}

In this section we will first reexamine in detail the result of the PPZ analysis and then present the results of our new analysis. As mentioned already, the PPZ analysis was based on the 1992 PDG data, improved by the latest (at that time) determination of the $\tau$ mass. In order to cancel out the dependence of the decay widths on some parameters (the pion mass, the quark mixing angle $U_{u d}$, the pion decay constant, the Fermi constant, and the muon or $\tau$ mass), PPZ considered only the ratios of the partial decay widths: $R_{\pi \mu}^{\pi e}, R_{\tau e}^{\mu e}$, and $R_{\tau \mu}^{\tau e}$. They did not take into account radiative corrections for these processes under the assumption that the radiative corrections of order of several percents are of no importance (note that the radiative corrections do not cancel each other in the ratios $R_{\pi \mu}^{\pi e}$ and $R_{\tau e}^{\mu e}$ ). The PPZ analysis was performed in the case of one massive neutrino $\left(\nu_{3}\right)$ and two almost degenerate very light $\left(m_{1} \sim m_{2} \ll m_{3}\right)$ ones. Their main result is that $\nu_{3}$ could have a mass in the interval

$$
155 \mathrm{MeV} \lesssim m_{3} \lesssim 800 \mathrm{MeV} \text {. }
$$

That is, all the 1992 PDG data could be fitted with $m_{3}$ in the above range. They then improved the upper limit in Eq. (16) by taking into account the constraint coming from the $Z$ invisible width. The resulting allowed interval was

$$
155 \mathrm{MeV} \lesssim m_{3} \lesssim 225 \mathrm{MeV} \text {. }
$$

Given the values of $m_{3}$ inside this range, the PPZ analysis also showed that one of the mixing angles (namely, $\beta$ ) was constrained to a finite range which did not include $\beta=0$. They obtained, for $m_{3}=165 \mathrm{GeV}$,

$$
11.54^{\circ} \lesssim \beta \lesssim 12.82^{\circ} \text {. }
$$

Although the above mass and mixing angle intervals are allowed by the ratios $R$, one must make sure that the same allowed ranges do not violate the experimental partial decay widths. This turns out to be the case for the mass range $m_{3} \gtrsim 215 \mathrm{MeV}$, as will be shown in Sec. IV A.

In the following we will examine what would happen to the above PPZ conclusions if (1) radiative corrections are taken into account, (2) the constraint from $\bar{\Gamma}$ 's (decay 


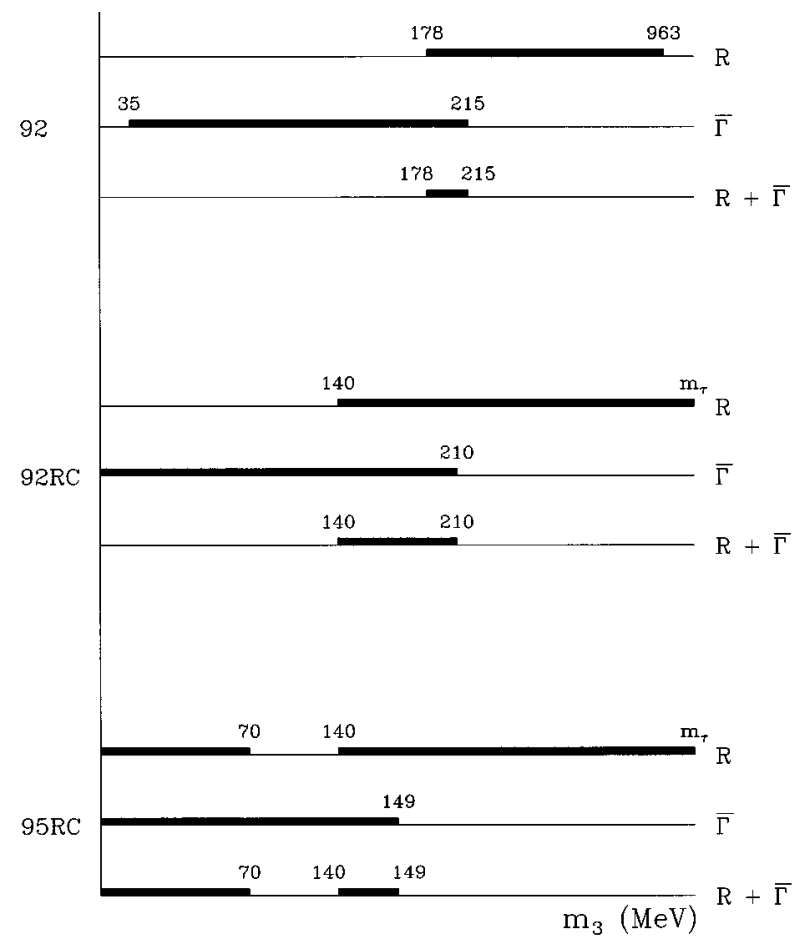

FIG. 1. Allowed (thick solid lines) and forbidden (solid lines) intervals of $m_{3}$ by $R$ 's, $\bar{\Gamma}$ 's, and $R$ 's and $\bar{\Gamma}$ 's combined, respectively, for 1992 and 1995 data sets $\left(m_{1} \simeq m_{2} \ll m_{3}\right.$ is assumed). Mixing angles are varied over the interval $(0, \pi / 2)$. The label 92 refers to the result of 1992 PDG data, without radiative corrections in the calculation; 92RC and 95RC denote the results of 1992 PDG and 1995 data sets with radiative corrections, respectively. The mass scale is in arbitrary units.

widths) is imposed, and (3) 1995 data are used together with the $\bar{\Gamma}$ constraint and the radiative corrections. Specifically, we will show that due to the accuracy of the present data, it is important to include radiative corrections and that the use of ratios $R$ 's alone without checking the partial decay widths (i.e., $\bar{\Gamma}$ 's) could lead to overestimates of the allowed interval of the neutrino parameters.

\section{A. Allowed range for mass}

In Fig. 1 we have plotted the ranges for the values of $m_{3}$ which are forbidden (denoted by solid lines) by the ratios $R$ 's, by the $\bar{\Gamma}$ 's, and by the combination of the two. The heavy solid line represents the allowed region. In this plot, it is assumed as in the case of the PPZ that the other mass parameters are very small $\left(m_{1} \simeq m_{2} \ll m_{3}\right)$ and the mixing angles are varied over the maximum interval $(0, \pi / 2)$. The figure refers to three cases: 1992 PDG data, without RC's, denoted by 92, 1992 PDG data with RC's, denoted by 92RC, and 1995 data with RC's, denoted by 95RC, respectively. For each case we present the three results, one with the $R$ 's, one with the $\bar{\Gamma}$ 's, and one with $R$ 's and $\bar{\Gamma}$ 's combined. For 92, there is an allowed region for $m_{3}$ which does not include $m_{3}=0$ when $R$ 's alone are used. This is the PPZ result. Most values of $m_{3}$ inside this allowed region, however, violate the limits on the $\bar{\Gamma}$ 's, as can be seen in Fig. 1. It has to be emphasized that the range for $m_{3}$ allowed by the $\bar{\Gamma}$ 's alone does not include $m_{3}=0$, also.
If we combine the two results, we find the following allowed range for $m_{3}$ :

$$
178 \mathrm{MeV} \lesssim m_{3} \lesssim 215 \mathrm{MeV} .
$$

The inclusion of radiative corrections changes the picture dramatically. As can be seen in Fig. 1 (92RC), the region allowed by $R$ 's is considerably enlarged. Also the $\bar{\Gamma}$ constraint is modified in an important way; i.e., the entire region of lower masses is now allowed, including $m_{3}=0$. When we combine the two constraints $(R+\bar{\Gamma})$ the PDG 92 data set is consistent with the following finite (not including zero) mass range for $m_{3}$ :

$$
140 \mathrm{MeV} \lesssim m_{3} \lesssim 210 \mathrm{MeV},
$$

signaling new physics beyond the standard model. It is, therefore, extremely interesting to repeat the analysis with the 1995 data. It is to be noted that the agreement of the above result with PPZ's [Eq. (17)] is purely accidental.

As can be seen in 95RC in Fig. 1, $R$ 's alone allow two different (disconnected) regions, one of which includes $m_{3}=0$. The inclusion of $\bar{\Gamma}$ 's restricts these intervals to the region of lower masses. The allowed regions resulting from the combined (both $R$ 's and $\bar{\Gamma}$ 's) analyses based on the 1995 data with radiative corrections are (for $m_{1} \simeq m_{2} \ll m_{3}$ )

$$
m_{3} \lesssim 70 \mathrm{MeV} \text { and } 140 \mathrm{MeV} \lesssim m_{3} \lesssim 149 \mathrm{MeV}
$$

The above result is rather insensitive to the choice of $m_{1}$ and $m_{2}$. For example, for $m_{1} \lesssim 20 \mathrm{keV}$ and $m_{2} \lesssim 1 \mathrm{MeV}$, the above result remains unchanged. Only if $m_{2}$ is of the order of a few $\mathrm{MeV}$ is the entire region between $m_{3}=0$ and $m_{3} \simeq 149 \mathrm{MeV}$ allowed. We note that the accuracy of the current data or even the 1992 PDG data warrants the inclusion of radiative corrections for any precision analysis. Furthermore, one can see that the use of the $R$ 's alone without the constraint from $\bar{\Gamma}$ 's can give rise to overestimated allowed regions.

\section{B. Allowed range for mixing angles}

So far we have reexamined and discussed PPZ's use of the 1992 PDG data. We have also carried out a similar analysis using the 1995 data with the conclusion that there is an allowed window for $m_{3}$ including $m_{3}=0$, as well as an isolated range of $m_{3}$ (for $m_{1} \simeq m_{2} \ll m_{3}$ ). In this subsection we carry out a similar analysis for mixing angles. PPZ conclude, based on the ratios alone from 1992 PDG data, that the angle $\beta$ has a finite allowed range $11.54^{\circ} \lesssim \beta \lesssim 12.82^{\circ}$, whereas the angle $\gamma$ is restricted to $\gamma \lesssim 4.05^{\circ}$, including zero. This allowed region is shown in the $\beta-\gamma$ plane as an area filled with circles in Fig. 2(a). In addition, the constraint imposed by the $\bar{\Gamma}$ 's alone is indicated by the dotted region. (It is to be pointed out that in Fig. 2 the mass parameters are $m_{1} \simeq m_{2} \simeq 0$ and $m_{3}=200 \mathrm{MeV}$.) The region allowed by the two constraints combined is denoted by the dark area.

Now, the inclusion of radiative corrections to the PPZ analysis leads to changes in the allowed regions of Fig. 2(a). The region allowed by $R$ 's alone is enlarged and moved towards the origin, but still it does not include the origin that corresponds to the case of neutrinos without mixing. This is 

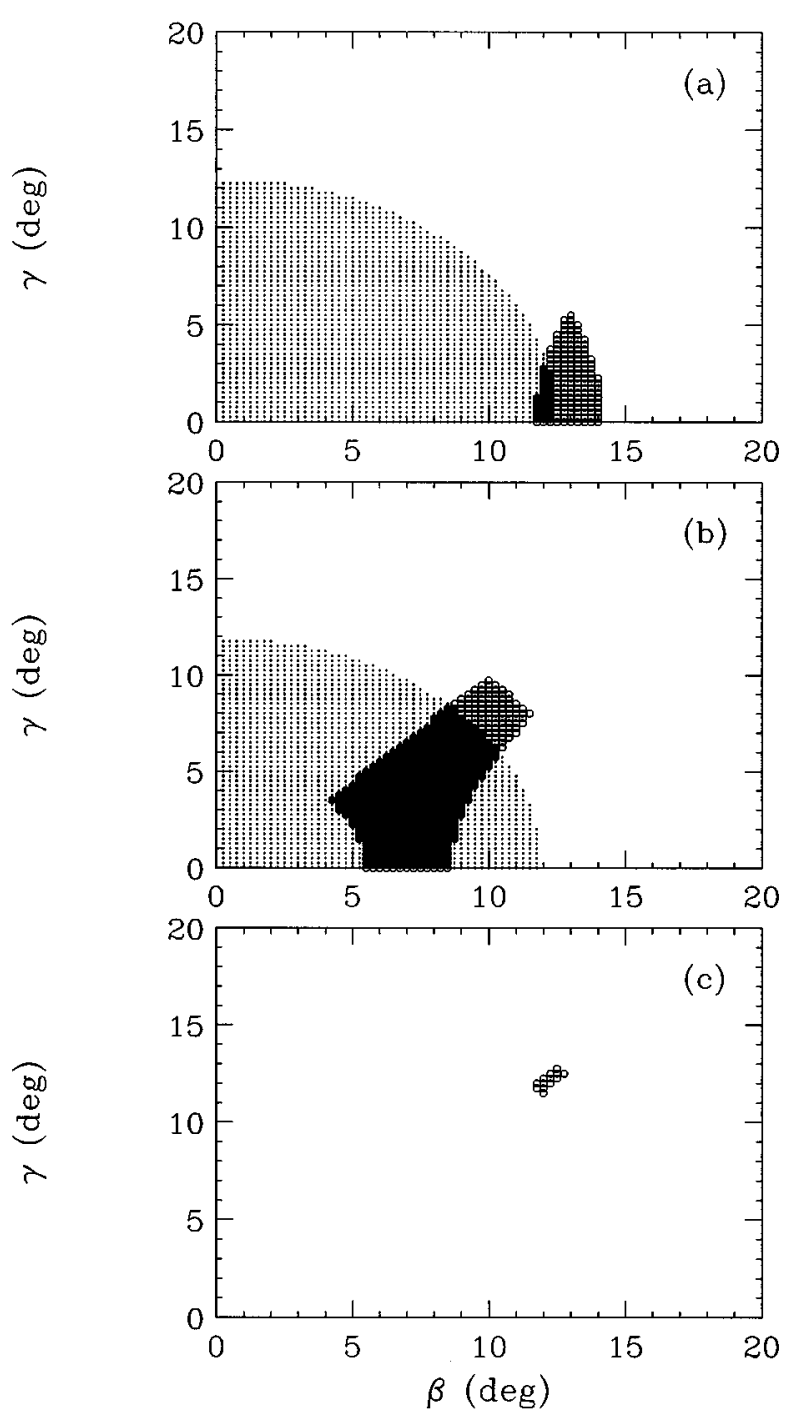

FIG. 2. Allowed (dotted) regions in the $\beta-\gamma$ plane for 1992 and 1995 data sets with $m_{1} \simeq m_{2} \simeq 0$ and $m_{3}=200 \mathrm{MeV}$ : (a) allowed region by $R$ 's alone (circles), $\bar{\Gamma}$ 's alone (dots), and $R+\bar{\Gamma}$ combined (dark area) of 1992 PDG data, (b) allowed region by $R$ 's alone (circles) and by $\bar{\Gamma}$ 's alone (dots) of 1992 PDG data with radiative corrections (the darker area is the region allowed both by the $\bar{\Gamma}$ 's and the $R$ 's), and (c) allowed region by $R$ 's alone (circles) of 1995 data with radiative corrections.

shown by circles in Fig. 2(b). Also shown in Fig. 2(b) is the allowed region based on $\bar{\Gamma}$ 's alone, with radiative corrections included (dots). The darker area is the region allowed by the $R$ 's and $\bar{\Gamma}$ 's combined. Therefore, the common allowed region is

$$
\begin{gathered}
4^{\circ} \lesssim \beta \lesssim 10^{\circ}, \\
\gamma \lesssim 7.2^{\circ} .
\end{gathered}
$$

When we use the 1995 data (with radiative corrections), the situation again changes dramatically. Figure 2(c) shows the allowed region in the $\beta-\gamma$ plane which is obtained by using the ratios $R$ alone (circles). The allowed region is shown to move farther away from the origin. The addition of the constraint coming from the $\bar{\Gamma}$ 's completely washes out the region, i.e., no allowed region. This is self-evident because the mass $m_{3}=200 \mathrm{MeV}$ is not allowed by the 1995 data, as can be seen in Eq. (21). Thus, even in the case of the 1995 data, neglecting the $\bar{\Gamma}$ constraint could lead to erroneous conclusions.

\section{ANALYSIS AND RESULTS}

In this section we present the results of a more detailed analysis of the limits that can be set on the neutrino masses and mixing angles by using the 1995 data and with the inclusion of radiative corrections. It is to be pointed out here that a complete, combined analysis of the masses $\left(m_{1}, m_{2}\right.$, and $\left.m_{3}\right)$ and mixing angles $(\theta, \beta$, and $\gamma)$ is very much involved and is beyond the scope of this paper. Even a presentation of the results of such an analysis would be problematic. Therefore, we have simplified the analysis by fixing some parameters and varying others. In order to see various correlations among the masses and the mixing angles, we present several allowed regions in two-dimensional plots for several combinations of mass and mixing angle.

First, we present the absolute upper limits on the three neutrino masses $m_{1}, m_{2}$, and $m_{3}$, independent of the values of mixing angles $\theta, \beta$, and $\gamma$. We have obtained these limits by varying the mixing angles over the entire interval between 0 and $\pi / 2$ and by taking into account the entire constraint which we have discussed in the previous section $(R$ 's and $\bar{\Gamma}$ 's) and the radiative corrections. (It should be stated that we are not carrying out a statistical analysis of all the relevant data.) Instead of three-dimensional plots, we present, in Fig. 3 , the allowed region (dotted area) in the $m_{1}-m_{2}$ plane and the allowed region in the $m_{2}-m_{3}$ plot in Fig. 4. From these plots, we can set the following absolute upper limits on the neutrino masses (based on $\bar{\Gamma}$ and $R$ constraint, at the $1 \sigma$ level):

$$
\begin{aligned}
& m_{1} \lesssim 100 \mathrm{keV}, \\
& m_{2} \lesssim 7.5 \mathrm{MeV}, \\
& m_{3} \lesssim 149 \mathrm{MeV},
\end{aligned}
$$

where the limits on $m_{1}$ and $m_{2}$ are mainly due to the $R$ 's, whereas the limit on $m_{3}$ comes from the $\bar{\Gamma}$ constraint. The limit on $m_{3}$ has already been mentioned in the previous section. Although the limit on $m_{1}$ is rather poor, the limit on $m_{2}$ is larger by a factor of less than 30 than the latest limit from the kinematical analysis of the $\pi \rightarrow \mu+\bar{\nu}_{\mu}$ decay. Similarly, the limit on $m_{3}$ is larger only by a factor of 6 . It is quite interesting that the accuracy of the present data on the decay rates and branching ratios is already sufficiently good enough to set limits on $m_{2}$ and $m_{3}$ which agree, within one order of magnitude, with the results from a more involved kinematical determination. The important difference between the upper limits given in Eq. (24) and those in Eq. (1) is that the former is valid independently of mixing angles whereas the latter is valid only for the case of no mixing. Furthermore, the improvement in the data from 1992 is obvious from the conclusion that the upper limit on $m_{3}$ is set to 149 


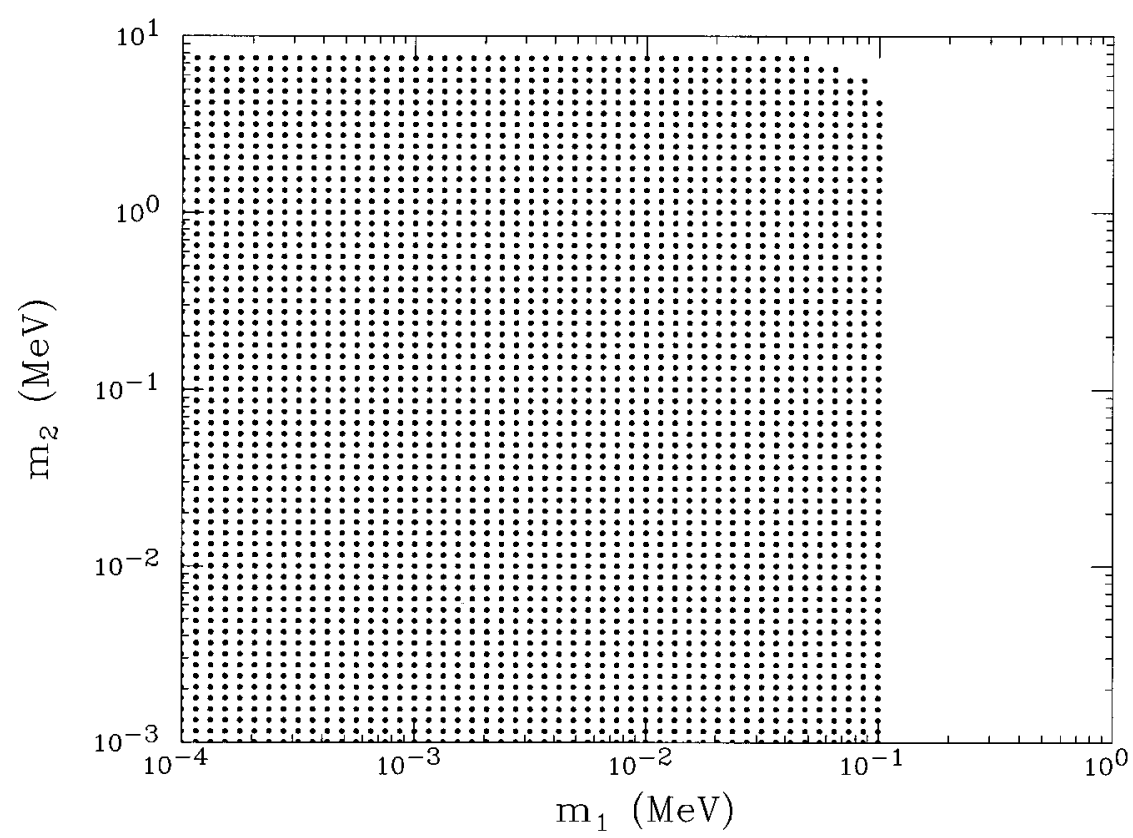

FIG. 3. Allowed (dotted) region in the $m_{1}$ $m_{2}$ plane for 1995 data with radiative corrections. Both $R$ 's and $\bar{\Gamma}$ 's constraints are imposed. Mixing angles are varied over the interval $(0, \pi / 2)$.
$\mathrm{MeV}$ and the limits are consistent with massless neutrinos with no mixing, implying the internal consistency of the data.

Next, we discuss some correlations among the masses and the mixing angles. The first example to be presented is the allowed region in the $m_{2}-\sin ^{2} \theta$ plot shown in Fig. 5. In this plot, we have set $m_{3}$ to be $24 \mathrm{MeV}$ and $\nu_{3}$ is assumed to be decoupled, due to its heavy mass, from $\nu_{1}$ and $\nu_{2}$ so that $\beta=\gamma=0$. Also, for definiteness, we have taken $m_{1}=5 \mathrm{eV}$, but the conclusion remains unchanged as long as $m_{1}$ is less than $\sim 20 \mathrm{keV}$. The solid and dashed lines delimit allowed regions based on the use of $\bar{\Gamma}$ 's and the constraint from $R$ 's, respectively. The allowed area is denoted by dots. Figure 5 shows that the low-angle regime is constrained mainly by $R_{\tau e}^{\mu e}$ whereas $R_{\pi \mu}^{\pi e}$ is more effective in limiting the large- angle area (say, $\left.\sin ^{2} \theta \geq 10^{-4}\right)$. The allowed region shown in Fig. 5 is insensitive to values of $m_{1}$ and $m_{3}$, as long as they are $m_{1} \lesssim 20 \mathrm{keV}$ and $m_{3} \lesssim 50 \mathrm{MeV}$. The so-called small- and large-angle solutions of the solar neutrino deficit based on the Mikheyev-Smirnov-Wolfenstein (MSW) effect, which requires $m_{2}^{2}-m_{1}^{2} \simeq 6 \times 10^{-6} \mathrm{eV}^{2}, \quad \sin ^{2}(2 \theta) \simeq 7 \times 10^{-3}$ and $m_{2}^{2}-m_{1}^{2} \simeq 8 \times 10^{-6} \mathrm{eV}^{2}, \sin ^{2}(2 \theta) \simeq 0.6$, respectively, are well within the allowed region in this plot.

In the next example, we assume that $\nu_{1}$ is too light to couple with $\nu_{2}$ and $\nu_{3}$. That is, only $\nu_{2}$ and $\nu_{3}$ are mixed with angle $\gamma$. We have also set $m_{1} \ll m_{2}=270 \mathrm{keV}$. Figure 6 shows the allowed region in the $m_{3}-\sin ^{2} \gamma$ plane. Here, the small- and large-angle regions are constrained by $R_{\tau e}^{\mu e}$, whereas the intermediate region $\left(\sin ^{2} \gamma \sim 10^{-3}-10^{-2}\right)$ is con-

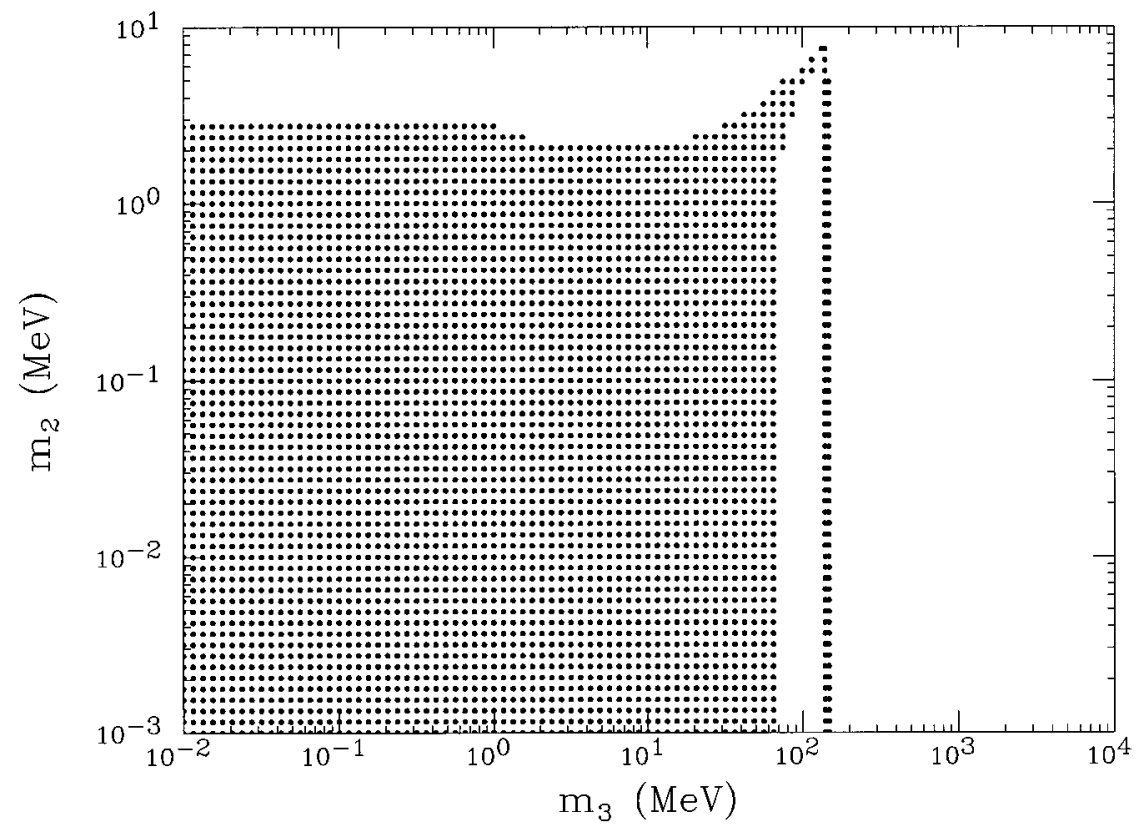

FIG. 4. Allowed (dotted) region in the $m_{2^{-}}$ $m_{3}$ plane for 1995 data with radiative corrections. Both $R$ 's and $\bar{\Gamma}$ 's constraints are imposed. Mixing angles are varied over the interval $(0, \pi / 2)$. 


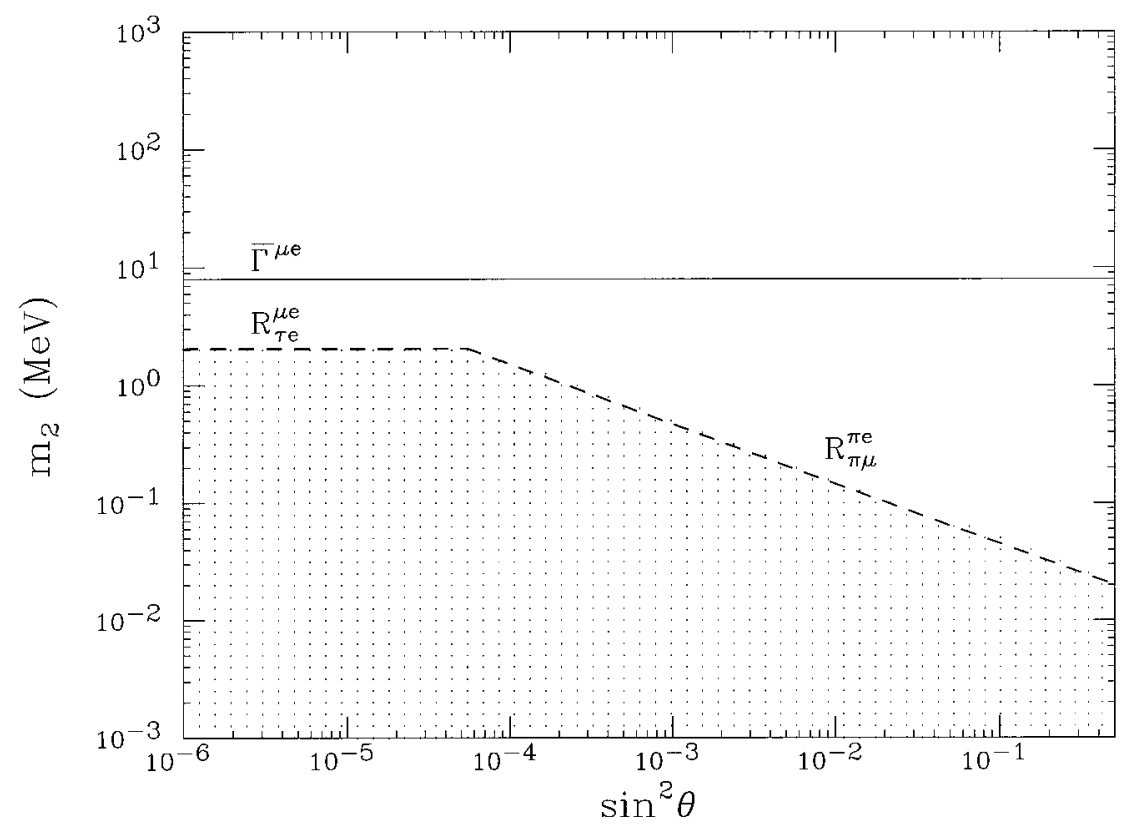

FIG. 5. Allowed (dotted) region in the $m_{2}$ $\sin ^{2} \theta$ plane for 1995 data with radiative corrections and $m_{1}=5 \mathrm{eV}, \quad m_{3}=24 \mathrm{MeV}, \quad$ and $\beta=\gamma=0$. The regions below the solid and the dashed lines are allowed by $\bar{\Gamma}$ 's alone and $R$ 's alone, respectively. strained by $R_{\pi \mu}^{\pi e}$. The neutrino oscillation solution of the atmospheric neutrino anomaly as observed by Kamiokande and others, which favors $m_{3}^{2}-m_{2}^{2} \simeq 10^{-2} \mathrm{eV}^{2}$ and $\sin ^{2}(2 \theta) \simeq 1$, is well within the allowed region in Fig. 6 . The size and the shape of the allowed region are insensitive to the assumed values of $m_{1}$ and $m_{2}$ as long as $m_{1} \lesssim 20 \mathrm{keV}$ and $m_{2} \lesssim 1 \mathrm{MeV}$.

Although the case of a $\nu_{1}-\nu_{3}$ mixing is unnatural in the framework of the natural hierarchy of neutrino masses, we present it in Fig. 7. In this figure the allowed region in the $m_{3}-\sin ^{2} \beta$ plane is shown. Here, as in the previous case we have set $m_{1} \ll m_{2}=270 \mathrm{keV}$. In this case, the most restricted limit is always imposed by $R_{\pi \mu}^{\pi e}$. Again, the allowed region does not change significantly as long as $m_{1} \lesssim 20 \mathrm{keV}$ and $m_{2} \lesssim 1 \mathrm{MeV}$.
In the previous figures, Figs. 5-7, the limits were obtained in the special cases in which two of the three mixing angles were kept fixed at zero; i.e., only one pair of neutrinos is mixed. To show how sensitive these limits are to the fixed angles and correlations among the limits, we present in Figs. 8-10 the cross sections (with one angle fixed) of the three-angle parameter space for fixed values of the masses, i.e., $\sin ^{2} \gamma$ - $\sin ^{2} \beta$ in Fig. $8, \sin ^{2} \gamma$ - $\sin ^{2} \theta$ in Fig. 9, and $\sin ^{2} \beta-\sin ^{2} \theta$ in Fig. 10, respectively. In each figure, the values of the masses are fixed as $m_{1} \ll m_{2}=270 \mathrm{keV}$ and $m_{3}=24 \mathrm{MeV}$. It is interesting to note that for the above set of the masses, the most severely constrained angle is $\beta$, whereas the least constrained is $\gamma$. The limits are:

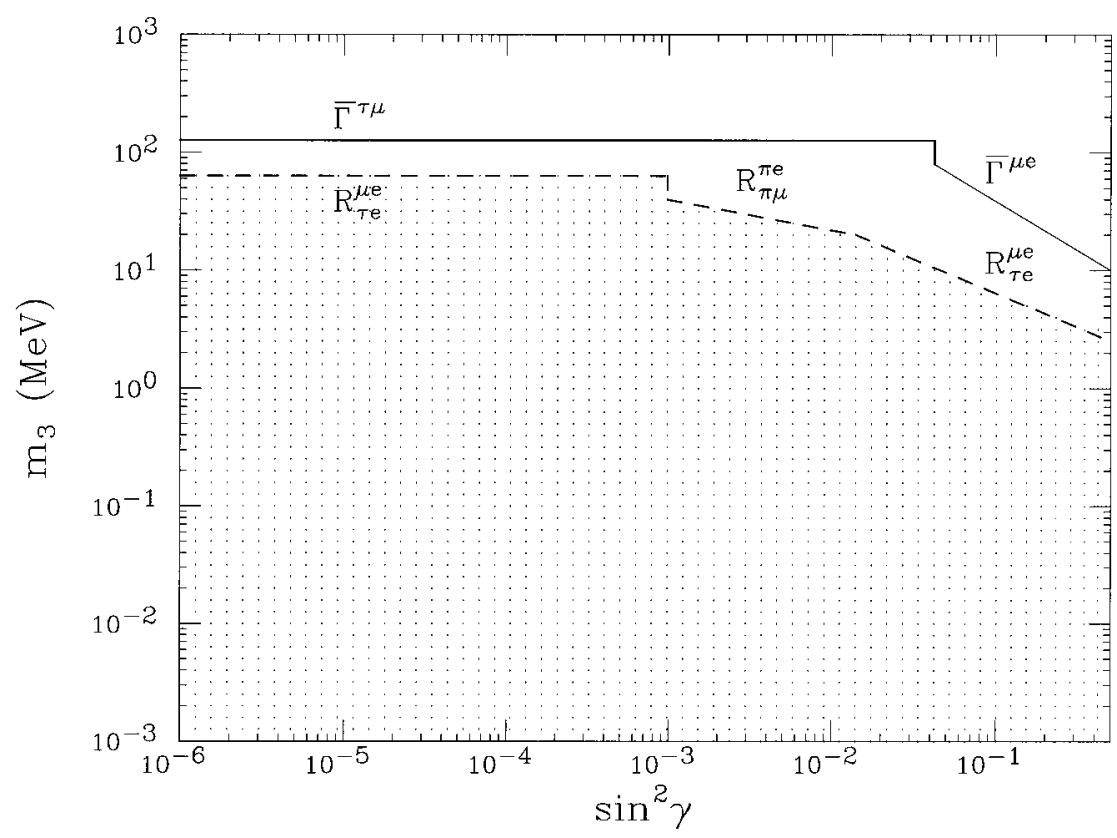

FIG. 6. Allowed (dotted) region in the $m_{3}$ $\sin ^{2} \gamma$ plane for 1995 data with radiative corrections and $m_{1}=5 \mathrm{eV}, \quad m_{2}=270 \mathrm{keV}$, and $\theta=\beta=0$. The regions below the solid and the dashed lines are allowed by $\bar{\Gamma}$ 's alone and $R$ 's alone, respectively. 


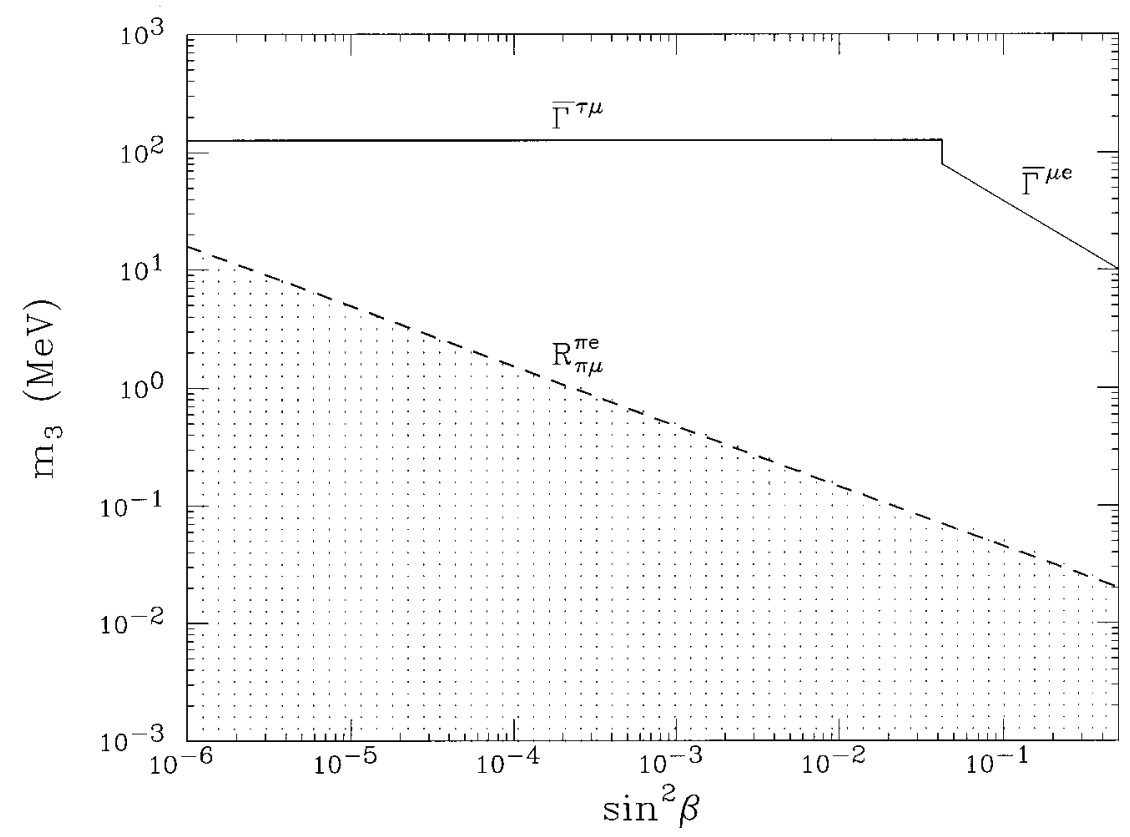

FIG. 7. Allowed (dotted) region in the $m_{3^{-}}$ $\sin ^{2} \beta$ plane for 1995 data with radiative corrections, and $m_{1}=5 \mathrm{eV}, \quad m_{2}=270 \mathrm{keV}, \quad$ and $\theta=\gamma=0$. The regions below the solid and the dashed lines are allowed by $\bar{\Gamma}$ 's alone and $R$ 's alone, respectively.

$$
\begin{aligned}
& \sin ^{2} \theta \lesssim 3.6 \times 10^{-3}, \\
& \sin ^{2} \beta \lesssim 4.6 \times 10^{-7}, \\
& \sin ^{2} \gamma \lesssim 7.0 \times 10^{-3} .
\end{aligned}
$$

As we decrease the values of the masses used, the allowed region in each figure increases, eventually covering the entire space. Hence, no meaningful limit can be obtained, as expected. In order to demonstrate this sensitivity, we have shown in Figs. 8-10 the extended allowed regions (bounded by the dashed lines) for the following values of the masses: $m_{1} \ll m_{2}=10 \mathrm{keV}$ and $m_{3}=1 \mathrm{MeV}$. In this case, the limits on the mixing angles substantially increase to

$$
\begin{gathered}
\sin ^{2} \theta \lesssim 1, \\
\sin ^{2} \beta \lesssim 2.6 \times 10^{-4}, \\
\sin ^{2} \gamma \lesssim 1 .
\end{gathered}
$$

\section{SUMMARY AND CONCLUSIONS}

We have analyzed and compared the 1992 and 1995 data on the $\pi, \mu$, and $\tau$ decays in the framework of three generations of massive neutrinos with associated mixing. First we have confirmed the surprising result of PPZ based on the 1992 PDG data that when only the ratios, $R$ 's, are used without radiative corrections, the 1992 data are inconsistent with the picture of massless neutrinos with no mixing, signaling

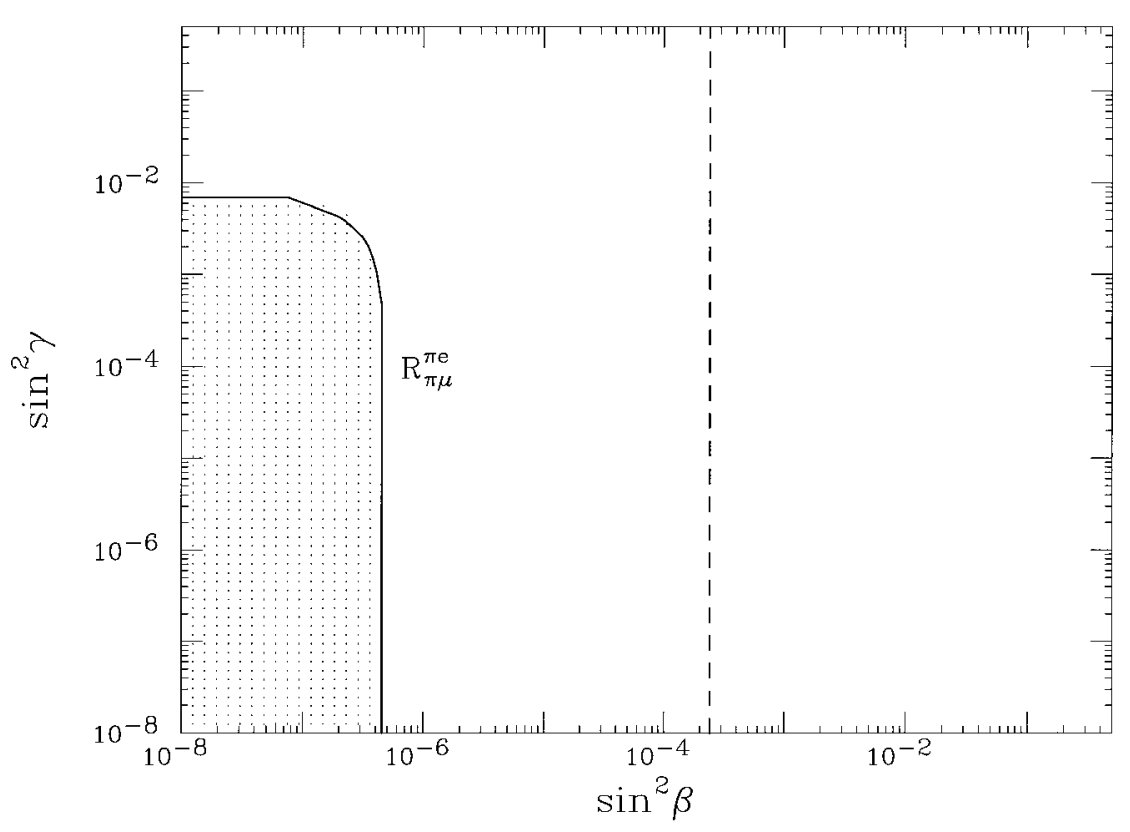

FIG. 8. Allowed regions in the $\sin ^{2} \gamma-\sin ^{2} \beta$ plane for 1995 data with radiative corrections. The dotted area inside the solid line is for $m_{1} \ll m_{2}=270 \mathrm{keV}$ and $m_{3}=24 \mathrm{MeV}$. The area on the left of the dashed line is for $m_{1} \ll m_{2}=10 \mathrm{keV}$ and $m_{3}=1 \mathrm{MeV}$. Note the enlargement of the allowed region as masses decrease. 


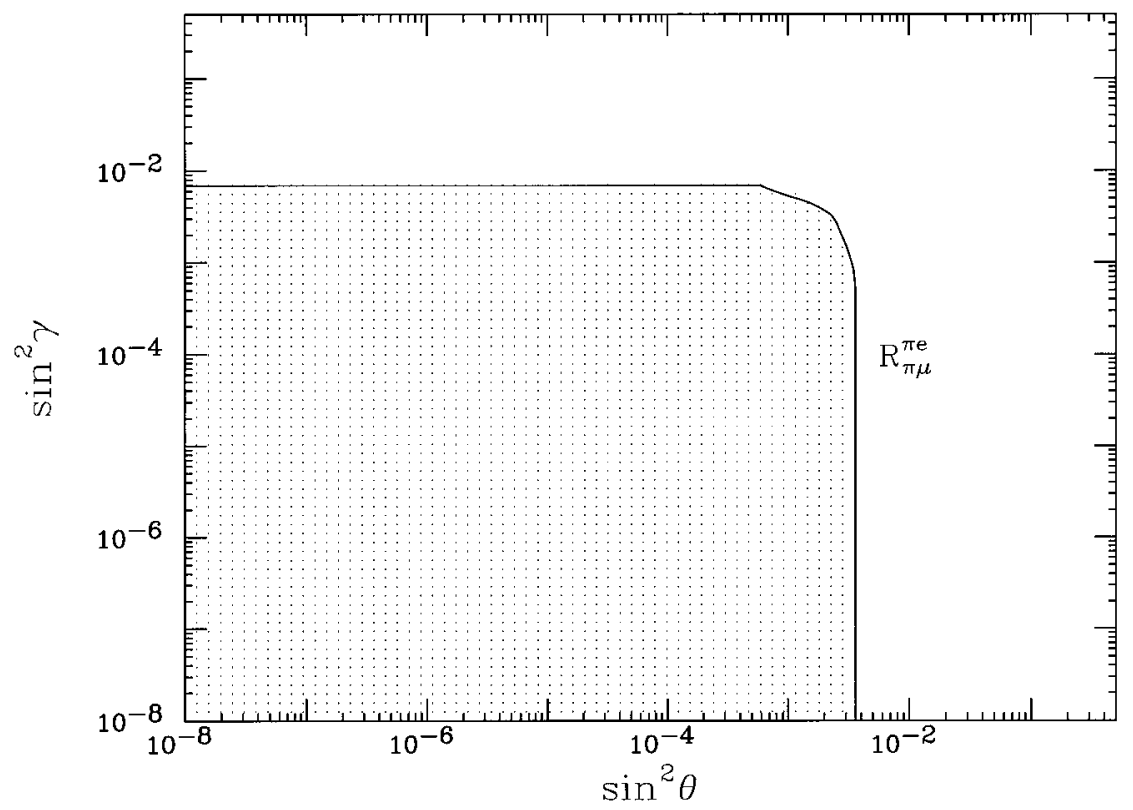

FIG. 9. Allowed regions in the $\sin ^{2} \gamma-\sin ^{2} \theta$ plane for 1995 data with radiative corrections. Notation and interpretation are the same as in Fig. 8.

new physics beyond the standard model. More specifically, PPZ have shown that when $m_{1}$ and $m_{2}$ are assumed to be much less than $m_{3}, m_{3}$ is found to be within the interval $155 \mathrm{MeV} \lesssim m_{3} \lesssim 225 \mathrm{MeV}$ and the $\nu_{1}-\nu_{3}$ mixing angle $\beta$ in the interval $11.54^{\circ} \leq \beta \leq 12.84^{\circ}$. This isolated allowed region survives even if we introduce the $\bar{\Gamma}$ constraint.

Again, the 1992 PDG data set with radiative corrections reproduces the results that agree qualitatively with those of PPZ. The allowed range of $m_{3}$ is $140 \mathrm{MeV} \lesssim m_{3} \lesssim 210 \mathrm{MeV}$ and the allowed mixing angles are $4^{\circ} \lesssim \beta \lesssim 10^{\circ}$ and $\gamma \lesssim 7.2^{\circ}$. This clearly shows that the 1992 PDG data set suggests massive and mixed neutrino.

In order to see if this rather surprising result still remains valid or not with the improved data of 1995, we have carried out a comprehensive analysis of the 1995 data by using both the ratios, $R$ 's, and decay widths, $\bar{\Gamma}$ 's, and by including the radiative corrections. The 1995 data are shown to be consistent with the picture of massless neutrinos with no mixing.

Limits on the masses derived from the analysis are

$$
\begin{aligned}
& m_{1} \lesssim 100 \mathrm{keV}, \\
& m_{2} \lesssim 7.5 \mathrm{MeV}, \\
& m_{3} \lesssim 149 \mathrm{MeV} .
\end{aligned}
$$

These bounds on the masses are such that the imposed constraint ( $R$ 's and $\bar{\Gamma}^{\prime} \mathrm{s}$ ) are fulfilled in their $1 \sigma$ intervals.

Although the above limits are less stringent than those in Eq. (1) from kinematical determinations, it is important to note that the limits given in Eq. (27) are completely independent of mixing angles. Therefore, if neutrinos are massive

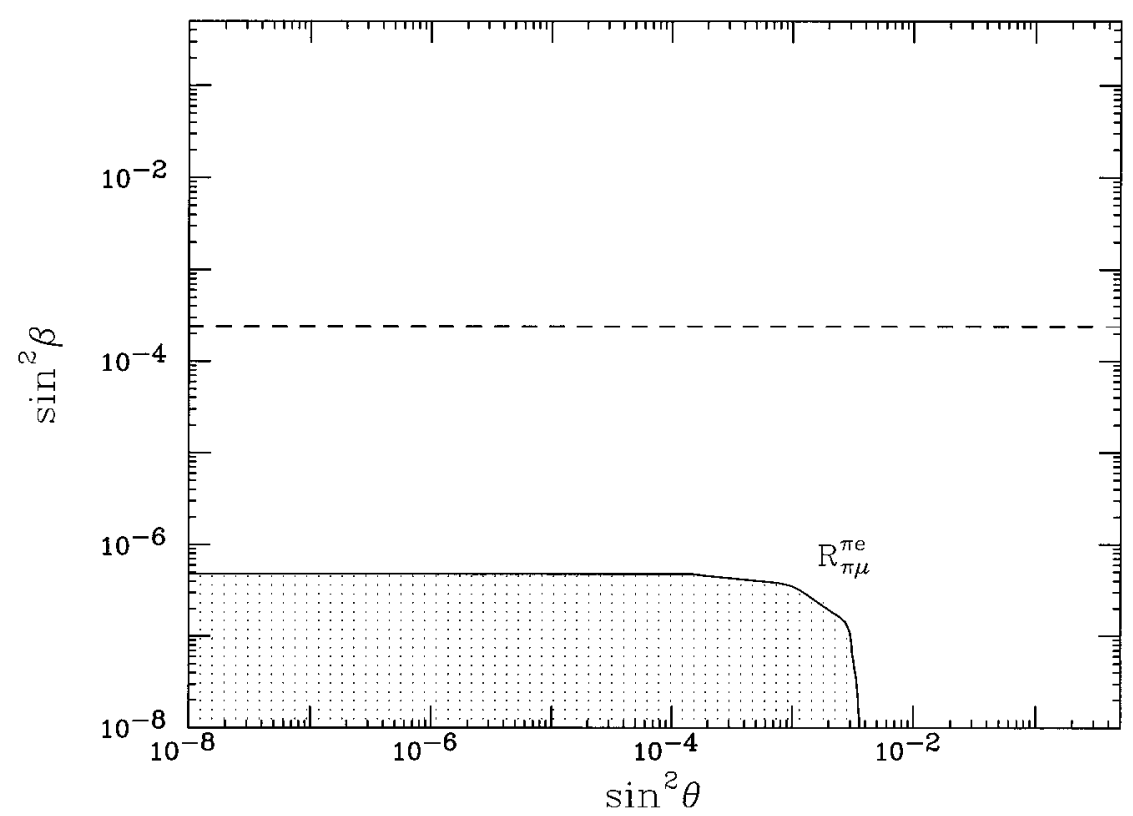

FIG. 10. Allowed regions in the $\sin ^{2} \beta-\sin ^{2} \theta$ plane for 1995 data with radiative corrections. Notation and interpretation are the same as in Fig. 8. 
and mixed, their masses can be heavier than the limits given in Eq. (1). Of course, such heavy neutrinos, if stable, are not allowed by the well-known cosmological limits $\sum_{i} m\left(\nu_{i}\right) \lesssim 20-30 \mathrm{eV} \quad$ [with $h=\left(H_{0} \mathrm{~s} \mathrm{Mpc}\right) / 100 \mathrm{~km} \simeq 0.5$, where $H_{0}$ is the Hubble constant]. If unstable, the decay of neutrinos must be such that it should not disturb the standard nucleosynthesis scenario and should not violate the observed limits on cosmic electromagnetic wave backgrounds.

It is not possible to obtain absolute limits on the mixing angles, because they strongly depend on the input values of the masses. Examples of limits on the angles for definite values of the masses have been derived and reported in the previous section.

To conclude, it is gratifying that the accuracy of the current data is already good enough to set limits on $m_{2}$ and $m_{3}$ which agree, within the order of magnitude, with the results from a more involved kinematical determination, although the limits on the mixing angles are still rather poor. Further improvements of the data on $\tau, m_{W}$, and $m_{Z}$ in the future may significantly improve the limits.

\section{ACKNOWLEDGMENTS}

The authors would like to thank B. Blumenfeld, J. Bagger, A. Falk, P. Gambino, L. Madansky, and G. Passarino for helpful discussions. Correspondence with O. L. G. Peres, V. Pleitez, R. Zukanovich Funchal, and R. E. Shrock was stimulating and very helpful. N.F. wishes to express his gratitude to the Fondazione "A. Della Riccia" for financial support and C.W.K. wishes to thank the Dipartimento di Fisica Teorica, Università di Torino, and INFN-Sezione di Torino for the hospitality extended to him where this work began. This work was supported in part by the U.S. National Science Foundation and by the Ministero della Ricerca Scientifica e Tecnologica, Italy.

\section{APPENDIX: FORMULAS FOR PION AND LEPTON DECAYS}

In this appendix we present, for completeness, the expressions for the pion and lepton decays for the general case of three massive neutrinos with mixing. Radiative corrections to these processes are included with a brief comment.

\section{Pion decay} by

The partial decay rate of the pion into two leptons is given

$$
\Gamma\left(\pi \rightarrow l \bar{\nu}_{l}\right)=\frac{G^{2} f_{\pi}^{2} U_{u d}^{2} m_{\pi}^{3}}{8 \pi} \mathcal{R}_{\pi l} \sum_{i=1}^{3}\left|V_{l i}\right|^{2} P_{i}^{\pi l},
$$

where the phase-space-matrix-element factor $P_{i}^{\pi l}$ is given by

$$
\begin{aligned}
P_{i}^{\pi l}= & \theta\left(m_{\pi}-m_{l}-m_{i}\right) \\
& \times\left[\delta_{l \pi}^{2}+\delta_{i \pi}^{2}-\left(\delta_{l \pi}^{2}-\delta_{i \pi}^{2}\right)^{2}\right] \lambda^{1 / 2}\left(1, \delta_{l \pi}^{2}, \delta_{i \pi}^{2}\right) .
\end{aligned}
$$

In the above, $G$ is the Fermi constant, $f_{\pi}$ is the pion decay constant, $U$ is the mixing matrix in the quark sector,

$$
\begin{gathered}
\delta_{l \pi}=\frac{m_{l}}{m_{\pi}}, \\
\delta_{i \pi}=\frac{m_{i}}{m_{\pi}} \quad(i=1,2,3),
\end{gathered}
$$

and $\lambda$ is the standard kinematical function,

$$
\lambda(x, y, z)=x^{2}+y^{2}+z^{2}-2(x y+y z+x z) .
$$

The quantity $\mathcal{R}_{\pi l}$ in Eq. (A1) describes the leading radiative corrections to the pion decay process $[4,16]$ given by

$$
\begin{aligned}
\mathcal{R}_{\pi l}= & {\left[1+\frac{2 \alpha}{\pi} \ln \left(\frac{m_{Z}}{m_{\rho}}\right)\right]\left[1+\frac{\alpha}{\pi} F\left(\delta_{l \pi}\right)\right]\left\{1-\frac{\alpha}{\pi}\left[\frac{3}{2} \ln \left(\frac{m_{\rho}}{m_{\pi}}\right)\right.\right.} \\
& \left.\left.+C_{1}+C_{2} \frac{m_{l}^{2}}{m_{\rho}^{2}} \ln \left(\frac{m_{\rho}^{2}}{m_{l}^{2}}\right)+C_{3} \frac{m_{l}^{2}}{m_{\rho}^{2}}+\ldots\right]\right\}
\end{aligned}
$$

where

$$
\begin{aligned}
F(x)= & 3 \ln x+\frac{13-19 x^{2}}{8\left(1-x^{2}\right)}-\frac{8-5 x^{2}}{2\left(1-x^{2}\right)^{2}} x^{2} \ln x \\
& -2\left(\frac{1+x^{2}}{1-x^{2}} \ln x+1\right) \ln \left(1-x^{2}\right) \\
& +2\left(\frac{1+x^{2}}{1-x^{2}}\right) L\left(1-x^{2}\right) .
\end{aligned}
$$

Here, $m_{\rho}=796 \mathrm{MeV}$ is the $\rho$ meson mass, $m_{Z}$ the $Z$ boson mass, and $\alpha$ is the fine-structure constant. Also, in the above, $L(z)$ is defined by

$$
L(z)=\int_{0}^{z \ln (1-t)} \frac{d}{t} d t
$$

The first set of square brackets in Eq. (A6) represents the electromagnetic short-distance correction. Its value is slightly modified when higher-order effects and QCD corrections are taken into account, i.e. [16],

$$
\left[1+\frac{2 \alpha}{\pi} \ln \left(\frac{m_{Z}}{m_{\rho}}\right)\right] \rightarrow 1.0232 .
$$

The second and the third sets of brackets denote the QED corrections to the decay of a pointlike pion $[16,17]$. Following a general practice, we neglect the terms with the $C_{i}$ 's, whose numerical values have large uncertainties [16]. Therefore, we use the simplified expression

$$
\mathcal{R}_{\pi l}=1.0232\left[1+\frac{\alpha}{\pi} F\left(\delta_{l \pi}\right)\right]\left[1-\frac{3 \alpha}{2 \pi} \ln \left(\frac{m_{\rho}}{m_{\pi}}\right)\right] .
$$

Note that $\mathcal{R}_{\pi l}$ depends both on the pion and the lepton mass.

\section{Lepton decay}

The partial decay rate of a lepton into three leptons is given by 


$$
\Gamma\left(l^{\prime} \rightarrow l \bar{\nu}_{l} \nu_{l^{\prime}}\right)=\frac{G^{2} m_{l^{\prime}}^{5}}{192 \pi^{3}} \mathcal{R}_{l^{\prime}} \sum_{i, j=1}^{3}\left|V_{l^{\prime} i}\right|^{2}\left|V_{l j}\right|^{2} P_{i j}^{l^{\prime \prime} l}
$$

where

$$
P_{i j}^{l^{\prime} l}=\theta\left(m_{l^{\prime}}-m_{l}-m_{i}-m_{j}\right) 2 \int_{x_{\min }}^{x_{\max }}\left(x^{2}-4 \delta_{l l^{\prime}}^{2}\right)^{1 / 2} \mathcal{M} d x
$$

and

$$
\mathcal{M}=\mathcal{A}^{1 / 2}\left[x\left(1+\delta_{l l^{\prime}}^{2}-x\right) \mathcal{A}+(2-x)\left(x-2 \delta_{l l^{\prime}}^{2}\right) \mathcal{B}\right],
$$

$$
\begin{aligned}
\mathcal{A}= & \frac{1}{\left(1+\delta_{l l^{\prime}}^{2}-x\right)^{2}}\left[\left(1+\delta_{l l^{\prime}}^{2}-x\right)^{2}-2\left(1+\delta_{l l^{\prime}}^{2}-x\right)\right. \\
& \left.\times\left(\delta_{i l^{\prime}}^{2}+\delta_{j l^{\prime}}^{2}\right)+\left(\delta_{i l^{\prime}}^{2}-\delta_{j l^{\prime}}^{2}\right)^{2}\right],
\end{aligned}
$$

$\mathcal{B}=\frac{1}{\left(1+\delta_{l l^{\prime}}^{2}-x\right)^{2}}\left[\left(1+\delta_{l l^{\prime}}^{2}-x\right)^{2}+\left(1+\delta_{l l^{\prime}}^{2}-x\right)\left(\delta_{i l^{\prime}}^{2}+\delta_{j l^{\prime}}^{2}\right)\right.$

$$
\left.-2\left(\delta_{i l}^{2},-\delta_{j l^{\prime}}^{2}\right)^{2}\right] \text {. }
$$

In the above

$$
\delta_{l l^{\prime}}=\frac{m_{l}}{m_{l^{\prime}}}
$$

$$
\begin{gathered}
\delta_{i l^{\prime}}=\frac{m_{i}}{m_{l^{\prime}}} \quad(i=1,2,3), \\
x_{\min }=2 \delta_{l l^{\prime}},
\end{gathered}
$$

$$
x_{\max }=1+\delta_{l l^{\prime}}^{2}-\left(\delta_{i l^{\prime}}+\delta_{j l^{\prime}}\right)^{2}
$$

The quantity $\mathcal{R}_{l}$, describes the leading radiative corrections to the lepton decay process [18] which are given by

$$
\mathcal{R}_{l^{\prime}}=\left[1+\frac{\alpha}{2 \pi}\left(\frac{25}{4}-\pi^{2}\right)\right]\left(1+\frac{3}{5} \frac{m_{l^{\prime}}^{2}}{m_{W}^{2}}\right),
$$

where $m_{W}$ is the $W$ boson mass. Note that $\mathcal{R}_{l^{\prime}}$ depends only on the mass of the decaying lepton, but not on the mass of decay products.
[1] R.E. Shrock, Phys. Lett. 96B, 159 (1980); Phys. Rev. D 24, 1232 (1981); 24, 1275 (1981).

[2] J. Bahcall and R.B. Curtis, Nuovo Cimento 21, 422 (1961).

[3] T. Yamazaki et al., in Proceedings of the International Conference on High Energy Physics, Leipzig, 1984, edited by A. Meyer and E. Wieckzorek (Akad. Wiss., Zeuthen, East Germany, 1984), p. 262.

[4] Particle Data Group, L. Montanet et al., Phys. Rev. D 50, 1173 (1994).

[5] F. Boehm and P. Vogel, Physics of Massive Neutrinos (Cambridge University Press, Cambridge, England, 1987); C.W. Kim and A. Pevsner, Neutrinos in Physics and Astrophysics, Contemporary Concepts in Physics Vol. 8 (Harwood Academic, Chur, Switzerland, 1993).

[6] ALEPH Collaboration, D. Buskulic et al., Phys. Lett. B 349, 585 (1995).

[7] O.L.G. Peres, V. Pleitez, and R. Zukanovich Funchal, Phys. Rev. D 50, 513 (1994).

[8] Particle Data Group, K. Hikasa et al., Phys. Rev. D 45, S1 (1992)
[9] BES Collaboration, J.Z. Bai et al., Phys. Rev. Lett. 69, 3021 (1992); ARGUS Collaboration, H. Albrecht et al., Phys. Lett. B 202, 211 (1992).

[10] L. Maiani, Phys. Lett. 62B, 183 (1976).

[11] A. Sirlin, Phys. Rev. D 22, 971 (1980); W.J. Marciano and A. Sirlin, ibid. 22, 2695 (1980).

[12] CDF Collaboration, F. Abe et al., Phys. Rev. Lett. 75, 11 (1995).

[13] CDF Collaboration, F. Abe et al., Phys. Rev. Lett. 74, 2626 (1995).

[14] P.B. Renton, in Proceedings of the XVII International Symposium on Lepton-Photon Interactions, Beijing, People's Republic of China, 1995 (unpublished); K. Hagiwara, ibid.

[15] H.S. Chen, in Proceedings of the XVII International Symposium on Lepton-Photon Interactions [14].

[16] W.J. Marciano and A. Sirlin, Phys. Rev. Lett. 71, 3629 (1993).

[17] T. Kinoshita, Phys. Rev. Lett. 2, 477 (1959).

[18] W.J. Marciano and A. Sirlin, Phys. Rev. Lett. 61, 1815 (1988). 